\title{
Fluoropolymer as dielectric in organic field effect transistor (OFET)
}

\begin{abstract}
Fluoropolymer is a type of dielectric material that have been used in fabricating Organic Thin Film Transistor (OTFT). Dielectric layer is one of the layers that make OTFT. It is the layer that sort the electric field that can be used. Fluoropolymer have unique characteristics that can be utilized for fabrication of OTFT. Fluoropolymer is low dielectric contact material which is needed in ultra-large-scale integration (ULSI) to breakdown the parasitic capacitance that affect the value of current flow. Drop cast technique has been used as depositing method on interdigitated electrode (IDE) on glass substrate. The length of the channel is varied to study the IV characteristic. Current- voltage (I-V) measurements have been used to measure the resistance at the different channel length. The value of resistivity can give some impact to the OFET device.
\end{abstract}

Keyword: Organic field effect transistor; Organic thin film transistor; Dielectric material; Fluoropolymer; Channel length; Resistance 